\title{
Structural positions and risk budgeting \\ Quantifying the impact of structural positions and deriving implications for active portfolio management
}

\author{
Ulf Herold*
}

* Ulf Herold is a quantitative analyst at Metzler Investment in Frankfurt/Main, Germany, and a Ph.D. student at the University of Frankfurt/Main, Germany.

Address: Dipl. Kfm. Ulf Herold, M.A. (WSU), Applied Research, Metzler Investment GmbH, Große Gallusstr. 18, 60311 Frankfurt/Main, Germany

Telephone: +49 692104 1104, Fax: +49 69 293136, Email: UHerold@Metzler.com

The author benefited from discussions with Prof. Raimond Maurer, University of Frankfurt/Main, Germany, and thanks Frank-Peter Martin and Nader Purschaker, both from Metzler Investment, Frankfurt/Main, Germany, for their support. 


\begin{abstract}
Structural positions are very common in investment practice. A structural position is defined as a permanent overweighting of a riskier asset class relative to a prespecified benchmark portfolio. The most prominent example for a structural position is the equity bias in a balanced fund that arises by consistently overweighting equities in tactical asset allocation. Another example is the permanent allocation of credit in a fixed income portfolio with a government benchmark.
\end{abstract}

The analysis provided in this article shows that whenever possible, structural positions should be avoided. Graphical illustrations based on Pythagorean theorem are used to make a connection between the active risk/return and the total risk/return framework. Structural positions alter the risk profile of the portfolio substantially, and the appeal of active management - to provide active returns uncorrelated to benchmark returns and hence to shift the efficient frontier outwards - gets lost. The article demonstrates that the commonly used alpha - tracking error criterion is not sufficient for active management. In addition, structural positions complicate measuring managers' skill.

The paper also develops normative implications for active portfolio management. Tactical asset allocation should be based on the comparison of expected excess returns of an asset class to the equilibrium risk premium of the same asset class and not to expected excess returns of other asset classes. For the cases, where structural positions cannot be avoided, a risk budgeting approach is introduced and applied to determine the optimal position size. Finally, investors are advised not to base performance evaluation only on simple manager rankings because this encourages managers to take structural positions and does not reward efforts to produce alpha. The same holds true for comparing managers' information ratios. Information ratios, in investment practice defined as the ratio of active return to active risk, do not uncover structural positions.

Keywords: active management; structural positions; information ratios; Pythagorean theorem; risk budgeting; tactical asset allocation

JEL classification: G11 


\section{Introduction}

In asset management, it is common practice to employ a two-stage procedure. The investor determines the strategic asset allocation (in a way to match his liabilities as good as possible) and thereby derives a benchmark which reflects this strategic positioning. The active portfolio manager manages the fund against this benchmark. ${ }^{1}$ The investor bears the risk of the strategic asset allocation, the portfolio manager is (only) responsible for the active risk he takes. $^{2}$ Both take two different points of view: the investor has a total return/total risk perspective, while the active manager moves within in an active return/active risk framework.

There has been a long debate about this "artificial" separation and whether the alpha tracking error criterion is sufficient for portfolio construction and performance evaluation in active portfolio management. In his well-known article, Roll (1992) addresses the issue that market indices, which serve as a proxy for the clients' benchmarks, are usually not efficient. ${ }^{3}$ The portfolio manager could improve on the Sharpe ratio by constructing a portfolio with the same total risk as the benchmark but a higher expected return by moving upwards from the benchmark portfolio to the efficient frontier. ${ }^{4}$ However, the alpha - tracking error criterion prevents him from doing so.

One shortcoming of the alpha - tracking error criterion is that the focus is on active risk only. This becomes a severe problem when the portfolio manager implements structural positions. The most prominent example for a structural position is the equity bias in a balanced fund induced by consistently overweighting equities (or, by overweighting equities more often than underweighting equities) in tactical asset allocation (TAA). Another example is the permanent allocation of credit in a fixed income portfolio with a government benchmark. Generally, a structural position can be defined as a permanent overweighting of a riskier asset class relative to a prespecified benchmark portfolio. This leads to a new reference point (e.g., a $20 \%$ exposure to credit or a $5 \%$ overweight in equities), from which the manager tactically deviates.

\footnotetext{
In this paper, the focus is on active management. Of course, the investor could also decide to employ a passive manager with the task to replicate the benchmark return.

2 The strategic asset allocation regularly accounts for over $90 \%$ of the total fund risk, as shown for example in Brinson et al. (1991).

3 If they were efficient, there would be no justification for active management, and investors will invest only in passive funds.
} 
The purpose of this paper is to quantify the impact of structural positions on the fund's total risk characteristics and to develop normative implications for portfolio management. The analysis provided in this article shows that whenever possible, structural positions should be avoided. The main reason is that structural positions dominate portfolio return and portfolio risk. They alter the portfolio's risk profile substantially and (usually) in a negative fashion from the investor's total risk/return perspective. The appeal of active management - to provide alpha, i.e. excess return uncorrelated to the benchmark return and hence to shift the efficient frontier outwards - gets lost. In addition, structural positions complicate measuring managers' skill.

To avoid structural positions, the investor can either amend the alpha - tracking error criterion by imposing an additional restriction on the fund's total risk, or he can demand from the manager to construct active portfolios that are orthogonal to the benchmark portfolio. The investment manager can avoid structural positions by altering his investment process for TAA decisions. He should compare his expected excess return forecast of an asset class with the equilibrium risk premium of that same asset class and not with expected excess return forecasts of other asset classes to derive active positions.

However in some cases, a structural position cannot be avoided. For instance, when the investor wants to partially shift from government bonds into corporate bonds, it would be necessary to replace the government bond benchmark by a broad benchmark. However, this might not be feasible because of lacking liquidity in the corporate segment or because of an insufficient portfolio size. The only way for the investor to get some exposure to credit is by allowing the manager to implement a structural position. It is crucial then to determine the optimal position size for this position. This can be done by using risk budgeting techniques. After quantifying the information ratio for a structural position, the appropriate active risk budget can be determined.

For performance evaluation, investors are advised not to compare managers according to their achieved active return only, because this encourages managers to take structural positions and does not reward efforts to produce alpha. Recently, investors have begun to base performance evaluation also on information ratios. However, information ratios, in investment practice

\footnotetext{
4 In this article, risk is defined as standard deviation, according to Markowitz (1959).
} 
defined as ratio of active return to active risk, do not uncover structural positions and therefore are prone to not measure managers' skill correctly.

This paper is organized as follows. In section one, we illustrate the implementation of a structural position using the case study of a balanced fund. We quantify the impact on total risk by making a connection between the active risk/active return and the total risk/total return framework. This is done by mathematical expressions as well as graphical illustrations using Pythagorean theorem. Section one also explores and discusses the underlying theoretical foundations of structural positions. Section two develops implications for active portfolio management. We show how TAA investment processes could be altered in order to avoid structural positions. For the cases that structural positions cannot be avoided, we derive the position sizes of structural positions using a risk budgeting approach. The last section summarizes implications for performance evaluation.

\section{Quantifying the impact of structural positions}

In this section, we illustrate the impact of structural positions on total fund's risk and return characteristics by using mathematical expressions as well as Pythagorean theorem for triangles. In the first part, we briefly review Pythagorean theorem and show how it can be applied for illustration of risk. Next, we explain that structural positions lead to structural information ratios and compare them to real information ratios. Finally, we discuss the underlying theoretical foundation of structural positions.

\section{Using triangles for risk analysis}

According to Pythagorean theorem, the squared length of the hypotenuse equals the sum of the squared lengths of both cathetes in a right angled triangle, or $c^{2}=a^{2}+b^{2}$. In every triangle, the square of a triangle side $\mathrm{c}$ is equal to the sum of the squares of the sides a and $b$, less the double product of the sides and the cosine of the angle $\delta$ (see figure 1). In algebraic terms,

[1] $c^{2}=a^{2}+b^{2}-2 a b \cos \delta$. 


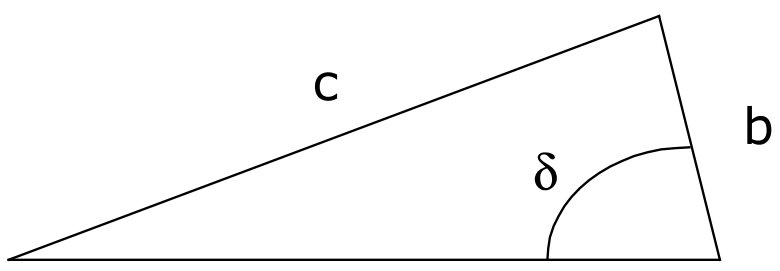

a

Figure 1

When comparing this equation to the equation for portfolio variance in the two asset case, [2]

$$
\sigma_{\mathrm{P}}^{2}=\mathrm{h}_{1}^{2} \sigma_{1}^{2}+\mathrm{h}_{2}^{2} \sigma_{2}^{2}+2 \mathrm{~h}_{1} \mathrm{~h}_{2} \rho_{12} \sigma_{1} \sigma_{2},
$$

where $h_{i}$ denotes the weight of asset $i, \sigma_{i}$ denotes standard deviation of asset $i$, and $\rho_{12}$ is the correlation coefficient between the two assets, the analogy becomes clear by setting $a=h_{1} \sigma_{1}$, $\mathrm{b}=\mathrm{h}_{2} \sigma_{2}$, and $\delta=\arccos \left(-\rho_{12}\right)$. Portfolio risk and different kinds of risk decomposition can be illustrated by triangles.

For instance, the impact of a varying correlation coefficient on the risk of a portfolio investing in two asset classes is demonstrated in figure 2 . A portfolio is invested $25 \%$ in equities and $75 \%$ in bonds. The volatilities of equities and bonds are $20 \%$ and $4 \%$, respectively. The varying correlation between equities and bonds corresponds to a varying angle. The portfolio moves on a circle with radius 3 . If the correlation is 1 , portfolio volatility is $8 \%$ (the sum of the weighted standard deviations). If the asset classes are uncorrelated, the angle is $90 \%$, and portfolio volatility is $5.8 \%$. No calculator is necessary, just a ruler.

Figure 2

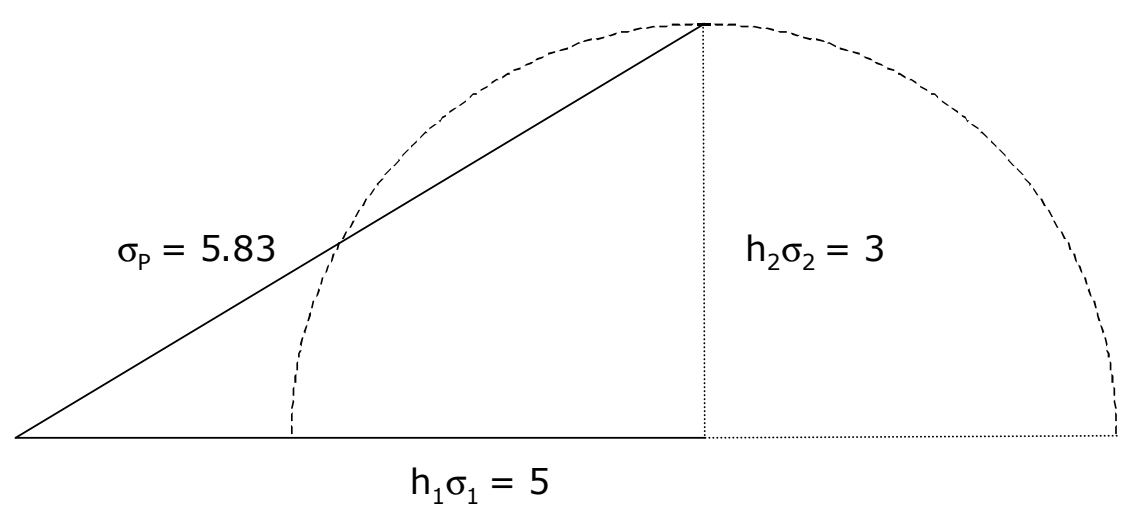

Another application is risk decomposition of the tracking error. The tracking error is a widely used expression for active risk. It measures the dispersion (volatility) of active returns around 
the mean active return. It consists of a systematic and a residual part. By dropping the perpendicular (see figure 3), the tracking error $\psi_{\mathrm{P}}$ is decomposed into its systematic component, $\beta_{\mathrm{PA}} \sigma_{\mathrm{B}}$, and its residual component, $\omega_{\mathrm{P}}$ :

[3] $\psi_{\mathrm{P}}^{2}=\beta_{\mathrm{PA}}^{2} \sigma_{\mathrm{P}}^{2}+\omega_{\mathrm{P}}^{2}$,

where $\beta_{\mathrm{PA}}$ is the portfolio's active beta and is defined as portfolio beta with the respect to the benchmark, $\beta_{\mathrm{P}}$, minus one.

Figure 3

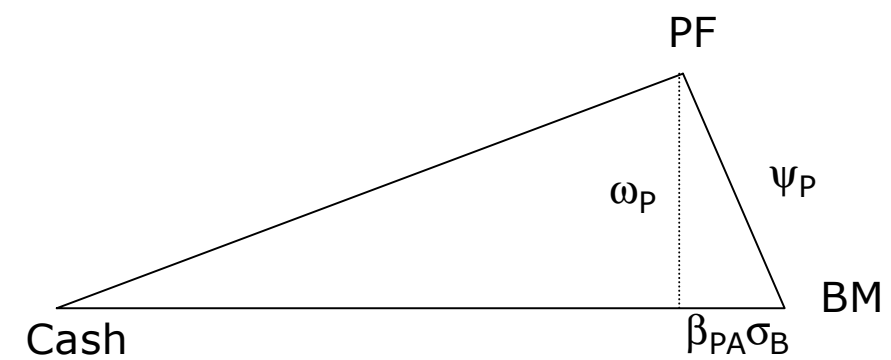

Figure 4 quantifies the risk contribution of active management on total risk. If active risk comprises only residual risk, the active portfolio is orthogonal to the benchmark portfolio. Total portfolio risk, $\sigma_{\mathrm{P}}$ (the line between PF and Cash), is only marginally higher than total benchmark risk, $\sigma_{\mathrm{B}}$ (the line between Cash and BM). For example, a balanced benchmark (stocks and bonds) might have a volatility of $12 \%$. Then a tracking error of $2 \%$ leads to a portfolio risk of $12.17 \%$. Hence figure 4 illustrates the appeal of active management in a graphical way: Active management produces orthogonal returns from which the investor fully benefits, while his risk profile is not altered substantially.

Figure 4

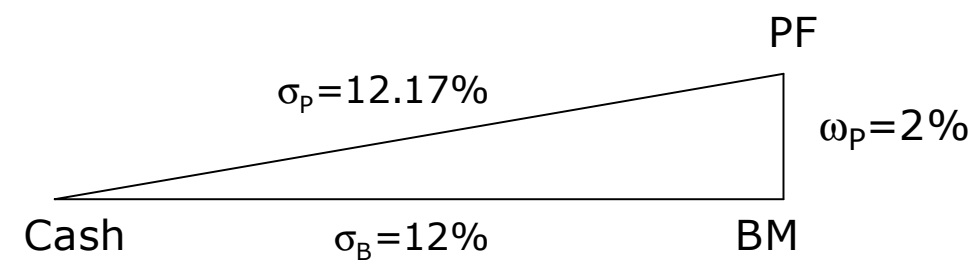

\section{Real versus structural information ratios - A case study}

Consider an investor who is invested in a balanced fund. The benchmark consists of $40 \%$ equities and $60 \%$ bonds. Volatilities $\sigma$ and expected excess returns $\mathrm{f}$ ( expected total returns 
over the risk-free rate) are shown in figure 5. The correlation between equities and bonds is assumed to be 0.2 .

Figure 5

\begin{tabular}{|c|c|c|c|}
\hline & $\mathrm{h}_{\mathrm{B}}$ & $\sigma$ & $\mathrm{f}$ \\
\hline $\mathrm{EQ}$ & 0.4 & $20 \%$ & $5.0 \%$ \\
\hline $\mathrm{FI}$ & 0.6 & $4 \%$ & $1.5 \%$ \\
\hline
\end{tabular}

This portfolio structure leads to an expected portfolio excess returns of $290 \mathrm{bp}$ for the investor. Portfolio volatility is $8.8 \%$, the (expected) Sharpe ratio, $\mathrm{f}_{\mathrm{P}} / \sigma_{\mathrm{P}}$, equals 0.33 . The investor wishes (or requires) a 50bp higher return. He asks an active manager to fulfill this task. The active manager can choose between two extreme positions (and any combinations, but we only consider the extreme cases in this section): he can either overweight equities, the asset class with higher expected excess returns, so much that expected portfolio excess return increases by $50 \mathrm{bp}$, without deviating from the benchmarks within the asset classes. Or he can do security selection within bonds and within equities in order to generate an alpha of 50bp but without shifting from bonds to equities. The first strategy is referred to as structural (as defined above): permanently overweighting the riskier asset class. We call the second way "real" active management.

The structural position will lead to an increased return of $50 \mathrm{bp}$, when overweighting equities by $14 \%$. The active risk following from this decision amounts to $280 \mathrm{bp} .^{5}$ This results in an information ratio of 0.18 . This information ratio is of structural nature because it simply results from shifting the asset classes.

Suppose the manager has a real information ratio (i.e., an information ratio based on selection skills) of equal size. Then he can generate an alpha of 50bp with an equal size of active risk. So far both strategies lead to the same result. The difference becomes clear when leaving the active return/active risk space. With the first strategy (structural position), portfolio total risk substantially increases from $8.8 \%$ to $11.3 \%$. The Sharpe ratio declines from 0.33 to 0.30 . In contrast, the second strategy achieves the same increase in return with only marginally rising portfolio volatility to $9.2 \%$, and the Sharpe ratio climbs to 0.37 .

\footnotetext{
5 Tracking error is calculated by substituting the vector of active weights, $\mathrm{h}_{\mathrm{PA}}$, into the standard deviation
} equation, $\left(\mathrm{h}_{\mathrm{P}}{ }^{\prime} \mathrm{V} \mathrm{h}_{\mathrm{P}}\right)^{0.5}$, where $\mathrm{V}$ is the (full) covariance matrix. 
Both strategies can be compared using the graphical tools introduced above. In figure 6, the portfolio resulting from the real active management strategy, denoted by $\mathrm{PF}^{\mathrm{r}}$, is orthogonal to the benchmark portfolio, because it is uncorrelated to the benchmark portfolio. The structural portfolio, $\mathrm{PF}^{\mathrm{s}}$, however, lies on the right side of the circle. (The circle comprises all active portfolios with a tracking error of $280 \mathrm{bp}$.) The angle $\delta$ can be determined by using a similar formula for the breakdown of risk as shown in figure 3 above. From

[4] $\sigma_{\mathrm{P}}^{2}=\sigma_{\mathrm{B}}^{2}+\psi_{\mathrm{P}}^{2}+2 \rho_{\mathrm{PA}, \mathrm{B}} \sigma_{\mathrm{B}} \psi_{\mathrm{P}}$,

it follows a correlation coefficient, $\rho_{\mathrm{PA}, \mathrm{B}}$, of 0.89 between the active structural portfolio and the benchmark portfolio. This very high correlation translates into an angle of $153^{\circ}$.

Figure 6

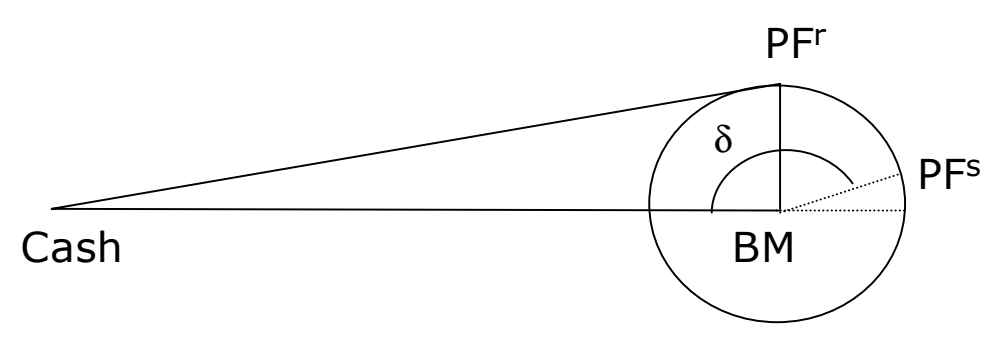

The distances between the points corresponding to cash and the two portfolios represent portfolio volatility. The volatility of the structural portfolio is much higher as it lies on the very right $-\mathrm{a}$ disadvantageous situation for the investor. The reason for this is that the structural portfolio has a beta of (much) greater than one. In our case study, using $\beta=\left(\mathrm{Vh}_{\mathrm{B}}\right) / \sigma_{\mathrm{B}}^{2}$, where $\mathrm{V}$ denotes the covariance matrix and $\mathrm{h}_{\mathrm{B}}$ is the vector of benchmark weights, yields a beta of 2.19 for stocks and a beta of 0.21 for bonds. This gives a beta coefficient of 1.28 for the structural portfolio. Using [3], it follows that about $80 \%$ of the tracking error variance of $(280 \mathrm{bp})^{2}$ is systematic.

There are different qualities of active return and active risk. A real active return, or alpha, is orthogonal to the benchmark return. Whatever the performance of the benchmark in a certain period, the alpha will stay the same (in the example, 50bp p.a.). In contrast, the structural active return is linked to a pretty high extent to the (direction and magnitude of the) benchmark return. Analogously, real active risk, or residual risk, has only a little effect on total risk, whereas structural active risk increases it substantially. 
The severe problem with the alpha - tracking error criterion is that it fails to consider the impact of active management on the client portfolio's level of total risk. It is located in the active risk/active return framework and does not allow any conclusions about total risk. Total risk, however, is the relevant measure for the investor. Active management can increase total risk by a great amount when implementing structural positions.

To summarize: A structural position leads to a structural information ratio. An investor will prefer a "real" information ratio to a structural information ratio when both are of equal size. The "real" information ratio arises from active positions which are uncorrelated to the benchmark portfolio so that total risk is only marginally increased. Structural positions lead to active portfolios which are highly correlated to the benchmark portfolio so that total risk is significantly increased. ${ }^{6}$

To avoid structural positions, the investor can either amend the alpha - tracking error criterion by imposing a constraint on the fund's total risk. Graphically, this constraint would be represented by a circle around the cash point. The manager's portfolio must lie within this circle. Or he can require that the active portfolio is orthogonal to the benchmark portfolio by imposing a beta constraint. However, this would prevent the manager from doing tactical asset allocation, which is an important source of value added in balanced funds. In section two, we will therefore discuss how the investment process could be changed in order to allow for tactical asset allocation but to avoid structural positions at the same time.

In addition to changing the fund's risk profile, structural positions complicate measuring manager's skill. It is difficult to disentangle the performance effect of structural positions from manager's skill. In the credit example from above, investors and managers will prefer broad fixed income benchmarks instead of gaining exposure to credit through structural offbenchmark positions: investors, because they can then better monitor the manager's performance, and managers, because they can demonstrate their value added more clearly. When managers are restricted to off-benchmark positions, they can only go long. Short selling is typically not permitted. This causes credit allocation to become an asymmetric decision (i.e., the manager can only implement bullish views), which lowers information ratio.

\footnotetext{
$6 \quad$ Structural positions will only reduce total risk, if the benchmark is located on the (inefficient) portion of the efficient frontier below the minimum variance portfolio. However, an investor will not choose an (ex ante) inefficient benchmark portfolio.
} 


\section{The underlying theoretical foundations}

An investor who believes that it is possible to beat the market and who has the skill to select good managers (with information ratios greater than zero before expenses) will invest in active funds. ${ }^{7}$ He will allow (or even urge) the manager to take residual risk to achieve alpha, although according to capital market theory, residual risk can be diversified and therefore offers no risk premium.

A portfolio manager implementing only structural positions assumes that the efficient market hypothesis (EMH) holds, ${ }^{8}$ and following CAPM and APT, he will not take any residual risk but simply shift into riskier assets to capture the higher risk premium associated with these assets.

The information ratio is usually employed to compare different managers or different strategies. The problem, however, is that in investment practice, the (expected) information ratio is regularly defined as expected active return divided by active risk $\left(\mathrm{f}_{\mathrm{PA}} / \psi_{\mathrm{P}}\right)$, whereas the correct definition, given in the literature, is alpha divided by residual risk $\left(\alpha_{\mathrm{P}} / \omega_{\mathrm{P}}\right){ }^{9}$ Alpha is defined as expected residual return (or risk-adjusted active return):

[5] $\quad \alpha_{P}=f_{P}-\beta_{P} f_{B}$.

This return decomposition corresponds to the risk decomposition provided in equation [3] above. Only if (portfolio) beta equals to one (or active beta equals to zero), alpha equals active return as well as residual risk equals active risk. As shown in the case study, an active beta arises due to structural positions. Hence, using only the information ratio (as defined by investment practice) is not sufficient.

Interestingly, when tracing the origins of active portfolio management, in their 1973 article, Treynor and Black proposed a three-stage procedure for the active management process: First, construct an active (long/short) portfolio that is uncorrelated to the market portfolio and maximizes information ratio. Second, combine this active portfolio in such a way with the market portfolio so that the resulting portfolio exhibits a maximum Sharpe ratio. And third, combine this portfolio and cash according to the investor's risk aversion.

Using a Bayesian framework, Baks et al. (2001) show that even an extremely skeptical prior belief in active managers' skill will lead to an economically significant allocation to active funds.

8 See Fama (1970) and Fama (1991).

9 See Grinold/Kahn (1999), Ch. 5. 
Hence, the objective of (real) active management is to produce alpha, i.e. active return that is uncorrelated to the benchmark return. Today, the investment practice focuses on the requirement to maximize information ratio, but has neglected, as least partly, the other requirements to produce an orthogonal active portfolio and to maximize the Sharpe ratio.

\section{Implications for portfolio management}

The analysis in section one has shown that structural positions should be avoided at all. The implication for the management of a balanced fund is not to change the benchmark and to keep away from structural positions by not overweighting equities in a permanent way (or, not "too often") to induce no equity bias. However, the question arises, how can a balanced fund manager nevertheless do tactical asset allocation? The first part of this section proposes how the investment process could be adapted in order to make tactical asset allocation work in a proper way.

For the second example, the inclusion of credit in a portfolio with a government bond benchmark, the implication is to replace the government benchmark by a broad benchmark consisting of government and corporate bonds (if this consistent with investor's preferences) and then manage actively against this broad benchmark. ${ }^{10}$ However, this might not be feasible because the liquidity of the corporate bond market may not (yet) be sufficient and/or the portfolio size may not be large enough. ${ }^{11}$ In these cases, the corporate index (as part of the broad index) cannot be replicated. The portfolio manager cannot achieve a neutral position from which he could deviate according to his market views. The only way for the investor to get a certain exposure to the credit market is to maintain the government benchmark but to allow the manager to implement structural positions in credit, which will contribute to the manager's active return and active risk. ${ }^{12}$ The manager has to decide which portion of the

10 Then the situation gets similar to the balanced fund example. When managing against a broad benchmark, the fixed income manager should not permanently overweight corporate bonds, otherwise he again introduces a structural position.

11 To replicate a corporate bond index, many more names must be included than in the case of a government bond index in order to ensure adequate coverage to systematic sectors and qualities. Also corporate bonds inherit much more specific risk (credit event risk) than government bonds, and specific risk can only be eliminated (or at least reduced) by diversification. Due to transaction costs and minimum trade sizes, diversification requires a minimum amount of at least 50 issuers, which in turn requires a certain minimum portfolio size.

12 Another reason in the past for not replacing a government bond index by a broad index was that no adequate broad indices existed (for Euroland). This has changed since the leading investment banks have expanded their well-accepted bond indices to include spread products. 
overall available active risk - the active risk budget - he assigns to this structural decision. ${ }^{13}$ In the second part of this section, we introduce risk budgeting techniques which provide a solution to this problem.

\section{Altering the investment process}

Tactical asset allocation (TAA) is an important source of value added in a balanced fund. As long as a manager has skill in TAA (i.e., positive real information ratio), he should do TAA. (The general procedure for deriving how much active risk he should take in a decision field like TAA is explained in the next section.) However, as section one has shown, shifting between asset classes which have very different risk levels easily produces undesirable structural positions. In practice, the TAA investment process is usually designed to compare expected excess returns for bonds and equities and base TAA on these forecasts. As the expected excess return for equities is higher than that for bonds in (far) more than $50 \%$ of all cases, this will lead the manager to overweight equities more often than underweighting equities. This, in turn, will produce a structural position.

To avoid this asymmetric outcome, the manager should compare his expected excess return for equities to the equity equilibrium risk premium (or, market consensus). ${ }^{14}$ If his forecast exceeds the equilibrium risk premium, he should overweight equities (and finance this overweight position out of cash, not out of bonds), otherwise he is advised to underweight equities and build up cash. Ex ante, the probability for the expected excess return to exceed the equilibrium risk premium is $50 \%$ (assuming a symmetric return distribution), so there is no structural bias in this decision any more. The same procedure should be applied for the bonds versus cash decision.

This procedure of basing TAA on (expected) deviations from equilibrium returns corresponds to Grinold and Kahn's approach for benchmark timing in a single asset class portfolio. For the portfolio's active beta, they show that the optimality condition holds:

13 In practice, the investor usually sets an upper limit for credit exposure (as well as lower bounds for rating categories) in the investment guidelines, but leaves it up to the manager to determine the position size within this range.

14 Of course, equilibrium risk premiums must be estimated, which introduces an additional source of estimation risk. On the other hand, this estimation risk should not be too large and not larger than the estimation risk associated with the manager's forecasts of expected returns. The estimation of equity risk premiums has been the subject of several studies; for recent studies see Pastor/Stambaugh (2000) and Jorion/Goetzmann (1999). 
[6] $\beta_{\mathrm{PA}}^{*}=\frac{\Delta \mathrm{f}_{\mathrm{B}}}{2 \lambda_{\mathrm{BT}} \sigma_{\mathrm{B}}^{2}},{ }^{15}$

where $\lambda_{\mathrm{BT}}$ is the investor's risk aversion to benchmark timing and

[7] $\Delta \mathrm{f}_{\mathrm{B}}=\mathrm{f}_{\mathrm{B}}-\mu_{\mathrm{B}}$

is the difference between the manager's forecast of benchmark (excess) return, $f_{B}$, and the "usual" level of benchmark (excess) return, $\mu_{B}$. If the manager expects that the asset class will do better than usual, then he will increase beta, and vice versa. Again, the manager bases his decision on comparing his forecast with the equilibrium return of the same asset class and not on comparing it with the expected return on cash or any other asset class. Note that active beta plays the same role in Grinold and Kahn's concept of benchmark timing as in our framework for TAA introduced in section one. An active beta that is positive on average introduces a structural position. The exposure to systematic market risk is higher than what the investor intended.

The next section introduces tools to perform an (active) risk decomposition. Structural positions dominate the portfolio's active risk level and composition if they are of considerable size. Their contribution to tracking error variance will quickly get very large, especially when they involve asset classes with very different risk levels, which is the case in TAA. Then there is not enough risk budget left for the manager to demonstrate his skill in doing stock and bond selection.

\section{Using risk budgeting techniques to derive position sizes}

In active management, there are several sources to add value. A global fixed income manager, for instance, engages in duration management, yield curve management, country allocation, currency allocation, and bond selection. All deviations from the benchmark in each of these decision fields will contribute to the fund's tracking error. However, active risk is a scarce resource that must be employed efficiently because the investor sets a tracking error target, which represents an upper bound for the manager. ${ }^{16}$

15 See Grinold/Kahn (1999), Ch. 19

16 From a theoretical standpoint, the optimal level of active risk taken by the manager should be determined by the investor's risk aversion and not by a fixed target (or target range) for active risk. Jacobs/Levy (1996) explain how the loss in utility can be quantified by pursuing the latter strategy. However in investment practice, it will often be easier for the investor to express the (maximum) level of active risk he is willing to take than his risk aversion. 
Intuitively, the manager will assign a greater active risk budget to those decision fields, where he has a higher confidence. ${ }^{17}$ This confidence level will depend on the manager's skill and experience as well as to the underlying asset class and the degree of information efficiency. ${ }^{18}$ The more value added he can generate with a certain decision field, the greater the risk budget he will allocate to this decision field. As the various decisions are not perfectly correlated to each other, he can make use of the power of diversification. ${ }^{19}$ The portfolio information ratio increases and he can generate a higher alpha for a given tracking error.

The procedure for deriving optimal risk budgets for various decision fields is to set up a straightforward optimization problem that can be expressed in mathematical terms. Let $\mathrm{IR}_{\mathrm{i}}$ be the ex ante information ratio of decision field $\mathrm{i}(\mathrm{i}=1 \ldots \mathrm{N}), \psi_{\mathrm{i}}$ the active risk of decision field $i$, and let $\lambda$ represent the investor's active risk aversion. ${ }^{20} \alpha_{\mathrm{P}}$ is the fund's active return and $\psi_{\mathrm{P}}$ its active risk. Value added, VA, is defined as

[8] $\mathrm{VA}=\alpha_{\mathrm{P}}-\lambda \psi_{\mathrm{P}}^{2}$.

Assume for the moment that the decision fields are uncorrelated to each other. Then, $\psi_{\mathrm{P}}^{2}=\sum_{\mathrm{i}} \psi_{\mathrm{i}}^{2}$. To maximize VA, take the first partial derivative of VA with respect to $\psi_{\mathrm{i}}$. This yields

[9] $\psi_{\mathrm{i}}^{*}=\frac{\mathrm{IR}_{\mathrm{i}}}{2 \lambda}$.

This equation clearly illustrates that only the information ratio is the relevant measure for deriving risk budgets. The belief that seems to dominate in investment practice is to take active risk in decisions (like the asset class decision in a balanced fund or the currency allocation in a fixed income portfolio) which have a great impact on portfolio performance due to the high volatility of the underlying assets. This turns out to be wrong.

17 In this section, we focus on active risk budgeting, as the budgeting of total risk is part of the strategic asset allocation. One issue of total risk budgeting is for the investor to determine how much of total risk should be assigned to active management. (Active management can be thought of as a separate asset class.)

18 Currencies, for instance, are very hard to predict. Hence, the (average) manager will not take too much risk in active currency positions.

19 Correlations do not refer to correlations between asset classes here, as it is the case in Markowitz's modern portfolio theory, but to correlations between the active returns of the various decision fields. Hence the diversification effect within the active risk budgeting framework is a different one from the usual diversification effect when talking about asset allocation.

20 To differentiate between total and active risk aversion, Clarke et al. (1994) use the term regret aversion for the latter. Regret aversion is generally much higher than total risk aversion. 
It is a first starting point to assume uncorrelated decision fields. As long as portfolio managers use different information sets for different decisions, there is no reason to expect positive or negative correlations. However, if they employ the same information set for certain decisions, e.g. duration and yield curve decisions, correlations will be positive. Correlations can be included in the optimization by introducing a $\mathrm{N} \times \mathrm{N}$ matrix $\rho$ containing the correlations between the decision fields. Let IR be the $N \times 1$ vector of the information ratios, $\psi$ be the $\mathrm{N} \times 1$ vector of the tracking errors, $\sigma$ be a $\mathrm{N} \times \mathrm{N}$ diagonal matrix with the tracking errors on the diagonal, and 1 be a $\mathrm{N} \times 1$ vector of ones. Then, equation [8] can be written in matrix notation: [10] $\mathrm{VA}=\mathrm{IR}^{\prime} \psi-\lambda 1^{\prime} \sigma \rho \sigma 1$.

This objective function can be maximized using numerical algorithms.

Total tracking error variance, $\psi_{\mathrm{P}}^{2}$, can be decomposed into risk contributions, or "contributions to active variance" (CAV). These CAVs represent the risk budgets; they sum up to tracking error variance. The CAVs can be standardized by dividing through $\psi_{\mathrm{P}}^{2}$. This gives the "absolute contributions to active variance" (ACAV). The N $\times 1$ vector of ACAVs can be calculated as follows:

[11] $\mathrm{ACAV}=\frac{\sigma \rho \sigma 1}{\psi_{\mathrm{P}}^{2}}$.

The risk budgeting approach can now be employed to derive the size of a structural position. The essence is that a structural position represents a further decision field that is in competition with the "traditional" decision fields (duration management, yield curve strategies, ...). The higher the (structural) information ratio of the structural position compared to the information ratios of the other decision fields, the more risk is allocated to the structural position. The procedure is to first calculate the structural information ratio (as done in section one), then perform the optimization. The optimization yields the optimal tracking error for the structural position, and from this number and the covariance matrix, the position size can be derived.

We illustrate this procedure with an example. Consider a fixed income manager with information ratios of $0.30,0.35$, and 0.10 for duration management, yield curve strategies and (government) bond selection (or relative value strategies), respectively. Duration and yield 
curve decisions are assumed to be correlated with 0.15 . The structural information ratio for credit is $0.25 .^{21}$ The optimization yields the risk budget distribution shown in figure 7 .

Figure 7

\begin{tabular}{|l|l|l|}
\hline & Information Ratio & Risk budget \\
\hline Duration management & 0.3 & $29.5 \%$ \\
\hline Yield curve strategies & 0.35 & $42.4 \%$ \\
\hline Relative value strategies & 0.1 & $3.9 \%$ \\
\hline Structural credit position & 0.25 & $24.3 \%$ \\
\hline
\end{tabular}

The largest risk budget is assigned to yield curve strategies, because it has the highest information ratio. The structural credit position receives a portion of almost $25 \%$ of the fund's active risk budget. If the investor targets an overall tracking error of $120 \mathrm{bp}$, the tracking error of the structural position amounts to $0.59 \%$. By using the covariance matrix V (covariances between the government and the corporate sector), the optimal position size amounts to $35 \%$. Hence, by allocating $35 \%$ of the portfolio into corporate bonds, the fund manager maximizes the portfolio information ratio.

\section{Conclusions}

In this paper, we have analyzed the link between the frameworks of total risk/total return and active risk/active return in the context of structural positions, which is a highly relevant topic in investment practice. Structural positions have disadvantageous effects for the investor. We have shown implications for portfolio construction and have derived techniques that can provide solutions.

Besides portfolio construction, the analysis has important implications for performance evaluation. In investment practice, many sponsors compare their managers (who are given the same benchmark) only on the basis of (realized) active returns, $\mathrm{r}_{\mathrm{PA}} \cdot{ }^{22}$ Managers are not only judged against their benchmarks but also against their peers. These one-dimensional rankings

21 Due to the longer available return history, the structural information ratio is based on US data. It is derived by using monthly return data from 3/1989 to 3/2001 for the Lehman US Corporate and the JPMorgan USA bond indices.

$22 \mathrm{r}$ denotes realized returns in contrast to the symbol $\mathrm{f}$ which represents expected (excess) returns (as used, for example, in equation [5]). 
are not very helpful to prevent managers from taking structural positions. In contrast, the opposite effect is true: They encourage or sometimes even force managers to engage in structural positions and do not reward efforts to produce alpha. ${ }^{23}$

Recently, more sponsors have recognized the drawbacks of simple manager rankings and amend the rankings by (realized) active risk and (realized) information ratios, $\mathrm{r}_{\mathrm{PA}} / \psi_{\mathrm{P}}$. However, as shown here, these measures (as defined in investment practice) are not able to detect structural positions. Hence, the investor is advised to go even further in order to ensure that the manager's strategy fulfills what the investor wants him to do (and pays him for): to produce excess returns uncorrelated to the benchmark and hence to shift the investor's efficient frontier outwards. $^{24}$

23 For instance, when all managers from the peer group managing a global fixed income portfolio with a world government benchmark do not allocate any JGBs, a single manager is more or less forced to also take this structural position.

24 This will of course increase the monitoring costs of the investor. If he is not willing to spend this effort, he is better advised to employ only single asset class managers and no balanced managers and to do the TAA by himself (or hire a single TAA overlay manager). 


\section{References}

Baks, K./Metrick, A./Wachter, J. (2001): Should all investors avoid all actively managed mutual funds? A study in Bayesian performance evaluation, in: Journal of Finance 56, No. 1, pp. $45-85$

Brinson, G./Singer, B./Beebower, G. (1991): Determinants of portfolio performance. An update, in: Financial Analysts Journal, May/June, pp. 40-48

Clarke, R./Krase, S./Stratman, M. (1994): Tracking errors, regret, and tactical asset allocation, in: Journal of Portfolio Management, Summer, pp. 16-24

Fama, E.F. (1970): Efficient capital markets: A review of theory and empirical work, in: Journal of Finance 25, No. 5, pp. 383-417

Fama, E.F. (1991): Efficient capital markets II, in: Journal of Finance 46, No. 5, pp. 15751617

Grinold, R./Kahn, R. (1999): Active Portfolio Management, 2nd edition, Chicago

Jacobs, B./Levy, K. (1996): Residual risk: How much is too much?, in: Journal of Portfolio Management, Spring, pp. 10-16

Jorion, P./Goetzmann, W. (1999): Global stock markets in the twentieth century, in: Journal of Finance, No. 3, pp. 953-980

Markowitz, H. (1959): Portfolio selection: Efficient diversification of investments, New York

Pastor, P./Stambaugh, R. (2001): The equity premium and structural breaks, in: Journal of Finance 56, forthcoming

Roll, R. (1992): A mean/variance analysis of tracking error, in: Journal of Portfolio Management, Summer, pp. 13-23

Treynor, J./Black, F. (1973): How to use security analysis to improve portfolio selection, in: Journal of Business, No. 1, pp. 68-86 